\title{
Supporting Information \\ Focal Plane Shift Imaging for the Analysis of Dynamic Wetting Processes
}

\author{
Hyeongyun Cha ${ }^{\dagger, \star}$, Jae Min Chun ${ }^{\dagger}$, Jesus Sotelo $^{\dagger}$, and Nenad Miljkovic ${ }^{\dagger, \star * *}$ \\ ${ }^{\dagger}$ Department of Mechanical Science and Engineering, University of Illinois \\ Urbana, Illinois 61801, USA \\ International Institute for Carbon Neutral Energy Research (WPI-I2CNER), Kyushu University, \\ 744 Moto-oka, Nishi-ku, Fukuoka 819-0395, Japan
}

*Corresponding Author E-mail: nmiljkov@illinois.edu 


\section{S1. Videos}

All videos were captured using a Phantom V711 Vision Research Camera. For surface fabrication and functionalization details, please see the methods section of the manuscript.

Video S1. Experimental top-view shifted-plane high speed video showing jumping droplet condensation of two droplets with radii $17.4 \pm 0.375 \mu \mathrm{m}$ and $17.4 \pm 0.375 \mu \mathrm{m}$ on a P2i coated horizontal superhydrophobic CNT surface. The video is captured with a 20X objective at a rate of $79,069 \mathrm{frames} / \mathrm{s}$, exposure time of $9.00 \mu \mathrm{s}$, period of $12.65 \mu \mathrm{s}$, and played back at 5 frames/s. The size of the image is $256 \times 256$ pixels, which represents $96 \times 96 \mu \mathrm{m}$.

Video S2. Experimental top-view in-plane high speed video showing jumping droplet condensation of two droplets with radii $5.63 \pm 0.375 \mu \mathrm{m}$, and $4.50 \pm 0.375 \mu \mathrm{m}$ on a P2i coated horizontal superhydrophobic CNT surface. The video is captured with a 20X objective at a rate of 210,526 frames/s, exposure time of $4.396 \mu \mathrm{s}$, period of $4.75 \mu \mathrm{s}$, and played back at 5 frames/s. The size of the image is $128 \times 128$ pixels, which represents $48 \times 48 \mu \mathrm{m}$.

Video S3. Experimental top-view in-plane high speed video showing jumping droplet condensation of two droplets with radii $25.9 \pm 0.375 \mu \mathrm{m}, 22.1 \pm 0.375 \mu \mathrm{m}$, and $19.9 \pm 0.375 \mu \mathrm{m}$ on a P2i coated horizontal superhydrophobic CNT surface. The video is captured with a $20 \mathrm{X}$ objective at a rate of 79,069 frames/s, exposure time of $9.00 \mu \mathrm{s}$, period of $12.65 \mu \mathrm{s}$, and played back at 5 frames/s. The size of the image is 256 x 256 pixels, which represents 96 x $96 \mu \mathrm{m}$.

Video S4. Experimental top-view in-plane high speed video showing jumping droplet condensation of four droplets with radii $8.25 \pm 0.375 \mu \mathrm{m}, 8.06 \pm 0.375 \mu \mathrm{m}, 7.88 \pm 0.375 \mu \mathrm{m}$, and $7.69 \pm 0.375 \mu \mathrm{m}$ on a P2i coated horizontal superhydrophobic CNT surface. The video is captured with a 20X objective at a rate of 210,526 frames/s, exposure time of $4.396 \mu \mathrm{s}$, period of $4.75 \mu \mathrm{s}$, and played back at 5 frames/s. The size of the image is $128 \times 128$ pixels, which represents $48 \times 48$ $\mu \mathrm{m}$.

Video S5. Experimental top-view in-plane high speed video showing jumping droplet condensation of two droplets with radii $57.4 \pm 0.375 \mu \mathrm{m}$ and $57.0 \pm 0.375 \mu \mathrm{m}$ initiated by droplet 
movement prior to droplet coalescence on a P2i coated horizontal superhydrophobic CNT surface. The video is captured with a 20X objective at a rate of 25,000 frames/s, exposure time of $15.0 \mu \mathrm{s}$, period of $40.0 \mu \mathrm{s}$, and played back at 5 frames/s. The size of the image is 512 x 512 pixels, which represents 192 x $192 \mu \mathrm{m}$.

Video S6. Experimental top-view in-plane high speed video showing jumping droplet condensation of six droplets with radii $10.50 \pm 0.375 \mu \mathrm{m}, 10.31 \pm 0.375 \mu \mathrm{m}, 10.31 \pm 0.375 \mu \mathrm{m}$, $7.31 \pm 0.375 \mu \mathrm{m}, 7.31 \pm 0.375 \mu \mathrm{m}$, and $5.63 \pm 0.375 \mu \mathrm{m}$ on a P2i coated horizontal superhydrophobic $\mathrm{CuO}$ surface. The video is captured with a 20X objective at a rate of 79,069 frames/s, exposure time of $12.292 \mu \mathrm{s}$, period of $12.65 \mu \mathrm{s}$, and played back at 5 frames/s. The size of the image is $256 \times 256$ pixels, which represents $96 \times 96 \mu \mathrm{m}$. 


\section{S2. Focal Plane Shift Imaging Procedure}

For each experimental trial, a set of strict procedures were followed to ensure the consistency throughout the experiments. To study jumping droplet condensation with Focal Plane Shift Imaging, we interfaced a top-view optical microscopy setup with a high-speed camera (Fig. S1, see Methods for details). To initiate condensation and droplet jumping, the superhydrophobic samples were horizontally mounted on a cold stage and cooled down to the test temperature of $T_{\mathrm{w}}$ $=1 \pm 0.5^{\circ} \mathrm{C}$ in a laboratory environment having an air temperature of $T_{\text {air }}=22 \pm 0.5{ }^{\circ} \mathrm{C}$ and a relative humidity of $\emptyset=28 \pm 1 \%$.

The First step of the process was to scan the surface with maximum window size $(1280 \times 800$ pixels) to find the appropriate droplet pair by horizontally moving the stage. Once droplets just prior to coalescence are observed, the window size was reduced to zoom in, and the framerate was increased. Depending on the droplet sizes, reduced window sizes ranging from 512 x 512 pixels to $32 \times 32$ pixels, and corresponding framerates ranging from 25,000 frames/s to 500,000 frames/s, were used. Then, the focal plane was placed in the middle of initial droplets where the clearest droplet edge could be observed (Fig. 2b), and the radii and location of the initial droplets was obtained.

The next step was to obtain the final conditions. The focal plane was manually elevated by vertically moving the z-stage down as far as the blurry images of the droplets through the elevated focal plane are visible (Fig. 2c). After shifting, we waited for the initial droplets to coalesce (2-5 seconds) and jump away from the surface. By limiting the focal plane shift distance to less than 10 times the diameter of the initial droplets, we ensured that the measured speed is indeed the initial jumping speed, and does not include the effects of droplet deceleration from vapor drag. As the merged droplet jumps from the surface and the center of the droplet passes through the shifted focal plane (Fig. 2d), location of the droplet and the total time from touch to crossing of the jumping droplet through the shifted plane were measured. The droplet time-of-flight was then calculated by subtracting the touch-to-liftoff time (see Section S3) from the total time measured from touch to crossing of the jumping droplet through the shifted plane. Note, if the initial droplets had not coalesced within short period of time, droplet radii might change, invalidating the results. In such cases, we re-shifted the focal plane back in the middle of initial droplets and replicated the experimental procedure again. 
After the blurry droplets disappeared (due to jumping), the elevated focal plane was shifted back to the surface to make sure that initial droplets were indeed removed from the surface (Fig. 2e). We also verified that only the identified droplets partook in the jumping process by observing the in-plane condensation re-nucleation behavior after jumping to ensure that droplets only formed at the pinned liquid bridges where prior droplets grew.

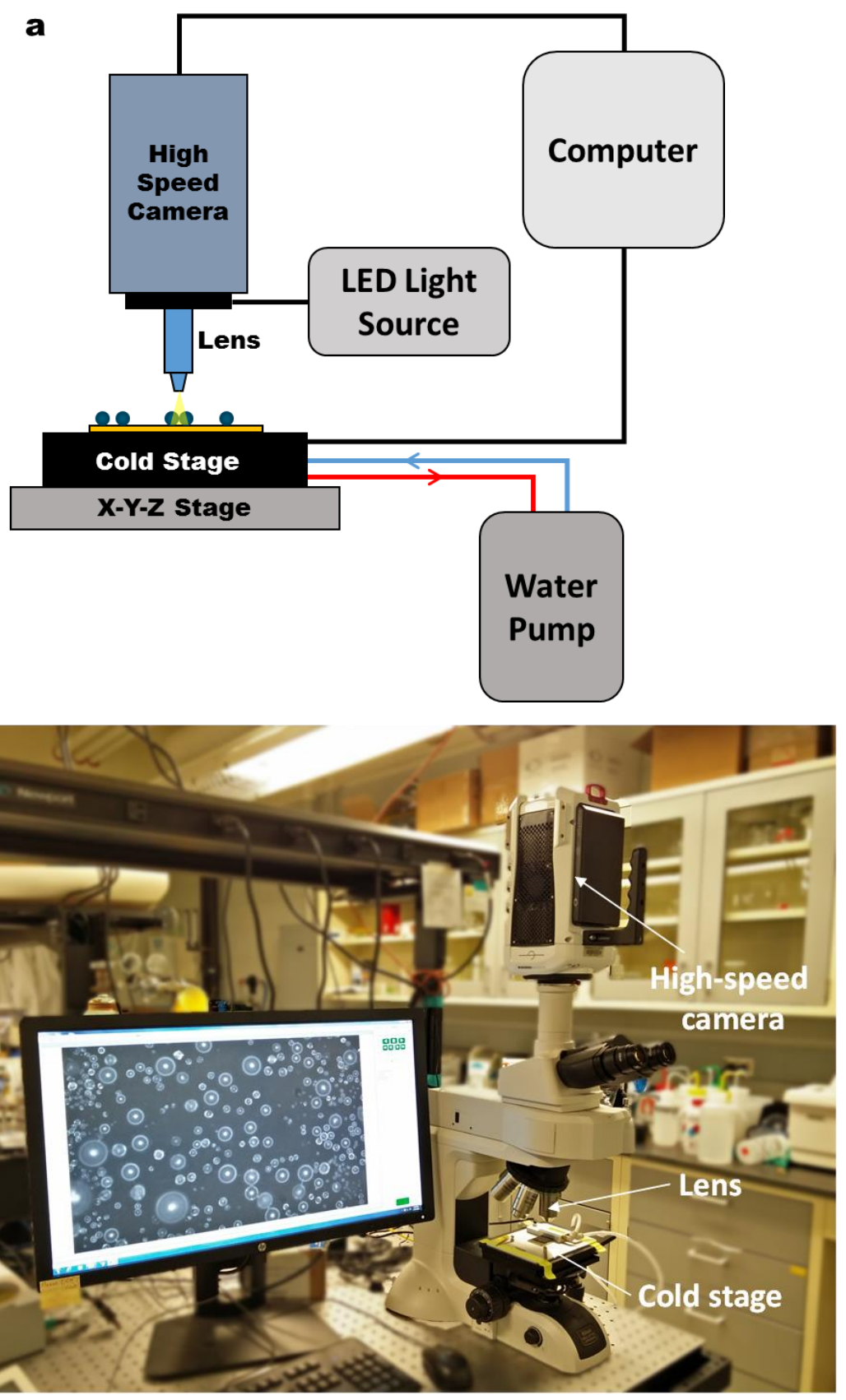

Figure S1. (a) Schematics of experimental setup (not to scale). (b) Photograph of experimental setup. 


\section{S3. Touch-to-Liftoff Time}

The out of plane speed of jumping droplets was calculated by dividing the droplet travel distance by the time-of-flight. The total time, $\tau_{\text {tot }}$, was first measured, defined as the time interval between the instant when droplets first touch, and when the jumping droplet passes through the elevated focal plane. However, $\tau_{\text {tot }}$ includes the time elapsed from droplet touch, to coalesced droplet liftoff. In order to measure the exact time-of-flight, $\tau_{\mathrm{TOF}}$, the time taken from droplet touch to liftoff, $\tau$, needs to be subtracted from the total time, i.e. $\tau_{\mathrm{TOF}}=\tau_{\text {tot }}-\tau$. To determine $\tau$, we experimentally measured the time taken between the instant droplet touch (Fig. S2 top images) and the instant droplet leave the surface (Fig. S2 bottom images) with in-plane high speed imaging. The liftoff state was determined by carefully observing when the coalesced droplet leaves the focal plane, signified be a diameter decrease (see Figs. 3 and 6a of the manuscript). Figure S3 shows $\tau$ as a function of departing droplet radius, $R_{\mathrm{j}}$, and number of coalescing droplets, $n$. The experimental results were fitted using the least square method to provide a reduced order model to be used in the focal plane shift imaging analysis.
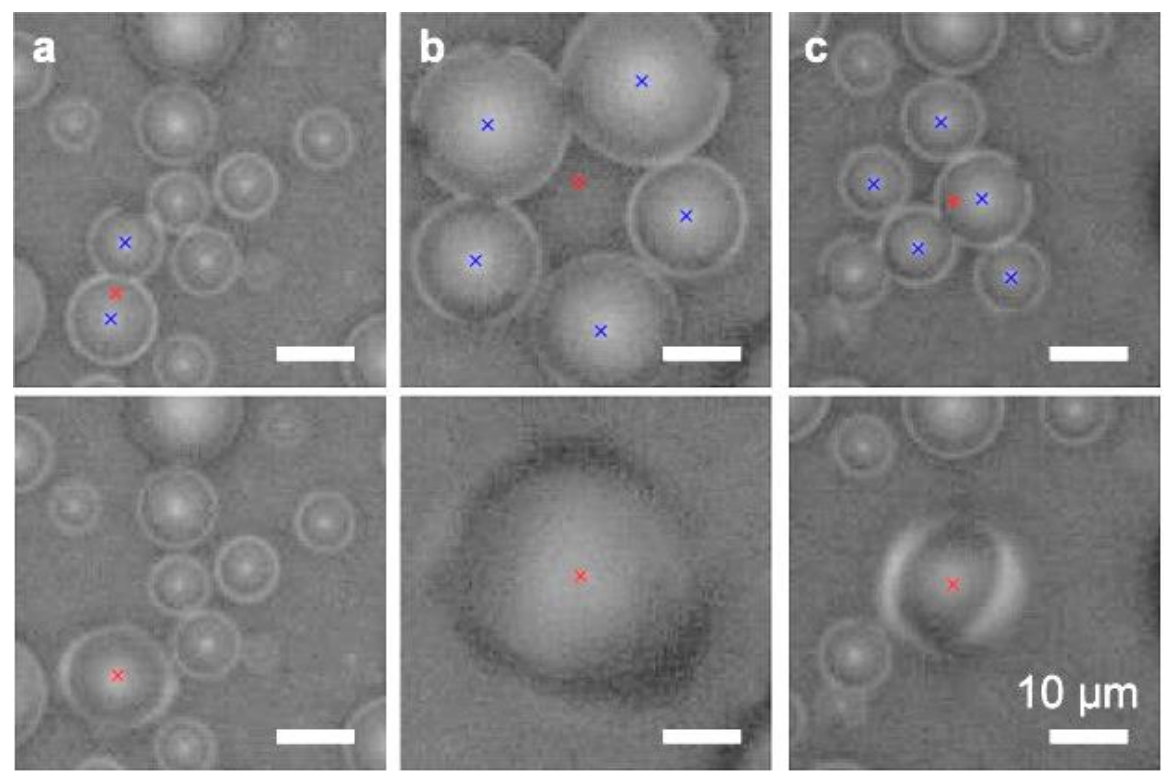

Figure S2. Top view in-plane high speed images of jumping droplet condensation. Each set of images shows the droplets just prior to coalescence (top) and just prior to jumping (bottom). Blue markers on the top images indicate the computed center of mass for each initial droplet prior to coalescence. Red markers on the top images indicate the computed center of mass for the merged droplet. Red markers on the bottom images indicate the computed center of mass just prior to jumping suing image analysis. The center of masses for the computed using initial conditions and post coalescence match very well, confirming that computed center of mass can be used as an initial location. All images were captured at a frame rate of 210,526 frames/s, exposure time of $4.396 \mu \mathrm{s}$, and period of $4.75 \mu \mathrm{s}$. 


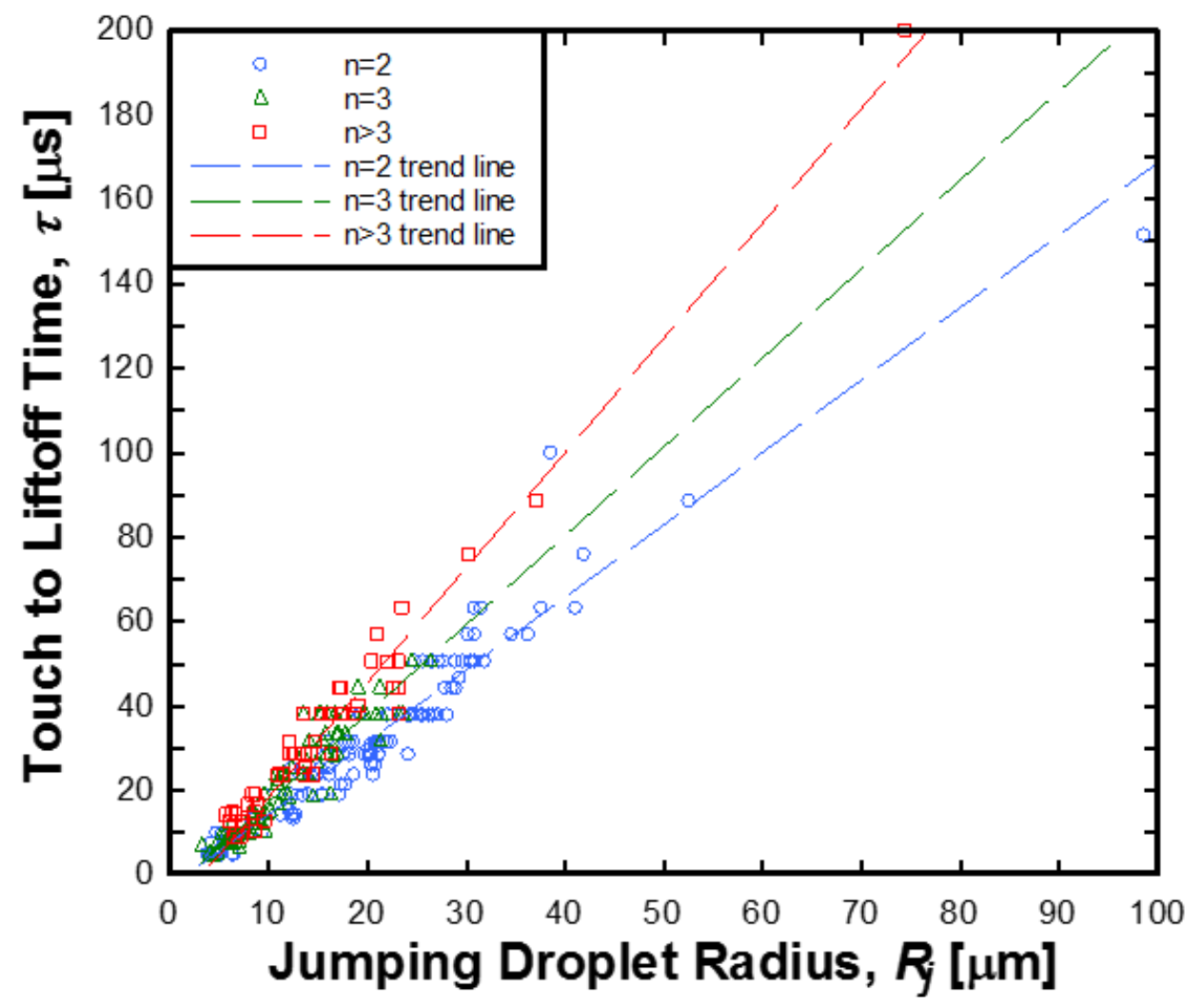

Figure S3. Touch-to-liftoff time, $\tau$, as a function of departing droplet radius, $R_{\mathrm{j}}$ for $n=2$ (blue circles), 3 (green triangles), and $>3$ (red squares). Each line shows the best fit line obtained using the least square method. The plotted results were used to compute the droplet travel time by subtracting $\tau$ from the total time. 


\section{S4. Initial Center of Mass}

The jumping droplet speed and departure angles were calculated by analyzing the initial and final location of the departing droplet. The final location of the droplet was defined as the location where the center of the jumping droplet passed through the elevated focal plane. The initial location of the jumping droplet was defined as the center of mass of the coalescing droplets, calculated from in-plane image analysis by applying the conservation of mass to the un-coalesced droplets (Fig. S2). Figure S2 shows time lapse images of in-plane droplet coalescence just prior to (top) and just after (bottom) coalescence. The blue markers on the top images represent the computed center of mass for each initial droplet just prior to coalescence using image analysis. The red markers on the top images represent the computed center of mass of the total system of droplets (blue markers). The red markers on the bottom images represent the computed center of mass just prior to jumping and after coalescence using image analysis. The images show that the computed centers of mass just prior to coalescence match well with the computed centers of mass using image analysis after coalescence (the red markers on the top images and bottom images are almost coincident). Furthermore, it was interesting to see that computed and measured centers of mass were independent of the number of droplets coalescing, as well as the size mismatch between droplets where Laplace pressure differences exist and drive flow asymmetrically. Intuitively, this result makes sense due to the absence of droplet-surface adhesion and lateral motion symmetry breaking. This fact is not true for droplet exhibiting significant pinning. It is also important to note that the computed center of mass using image analysis (red markers) also takes into account the zcomponent (out of plane direction). 


\section{S5. Independence of the Jumping Droplet Behavior on Surface Structure Length Scale}

To study if surface structure length scale has any effect on droplet departure speed, we analyzed the jumping droplet data. Figure $\mathrm{S} 4$ shows the jumping droplet speed, $U_{\mathrm{j}}$, as a function of departing droplet radius, $R_{\mathrm{j}}$, on different surfaces, and Figure S5 shows the polar angle, $\beta$, as a function of droplet size mismatch. The results show that the jumping droplet speed agrees well with inertialcapillary theory, and that the jumping droplet speed and polar angle are not correlated to the surface structure length scale. 

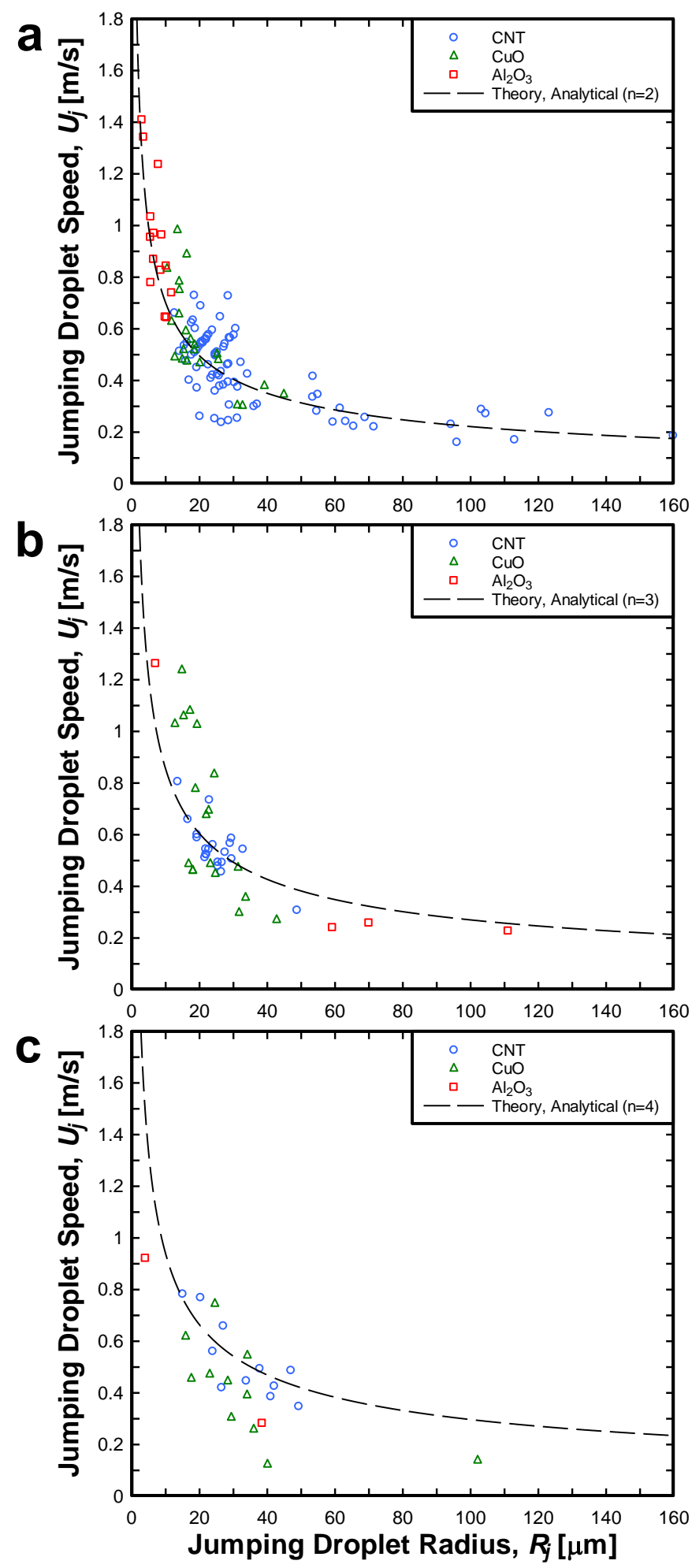

Figure S4. Experimental Jumping droplet speed, $U_{\mathrm{j}}$, as a function of departing droplet radius, $R_{\mathrm{j}}$, where the number of initial droplets, $n$, is (a) 2 , (b) 3 , and (c) $>3$, respectively. The results show that jumping droplet speed is not correlated to the surface structure. 

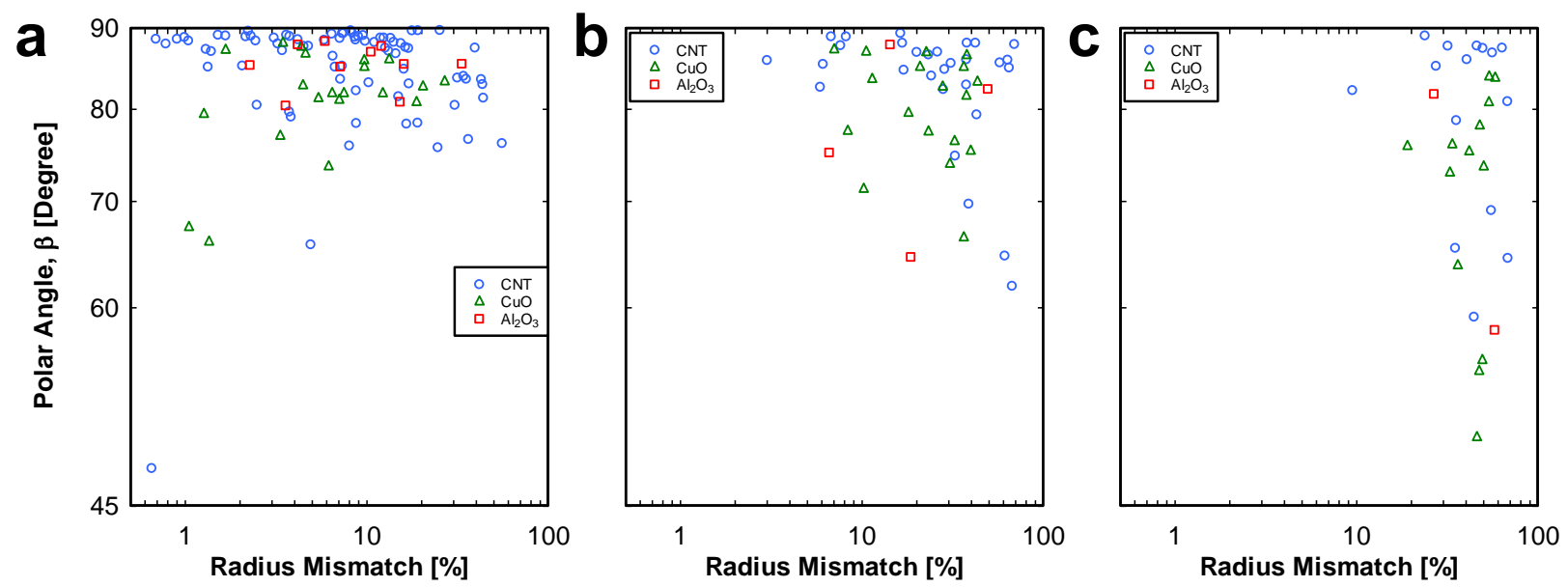

Figure S5. Polar angle as a function of initial droplet size mismatch where the number of initial droplets are (b) 2, (c) 3, and (d) $>3$. The results show that jumping droplet angle is not correlated to the surface structure. 


\section{S6. Independence of the Droplet Departure Behavior on Initial Droplet Arrangement}

To further study the effect of initial droplet arrangements on the droplet jumping behavior, we paid particular focus to the droplet coalescence and jumping with three droplets. Figure S6 shows the different arrangements of initial droplets just prior to jumping, including triangular, L-shaped, linear, and spaced configurations.
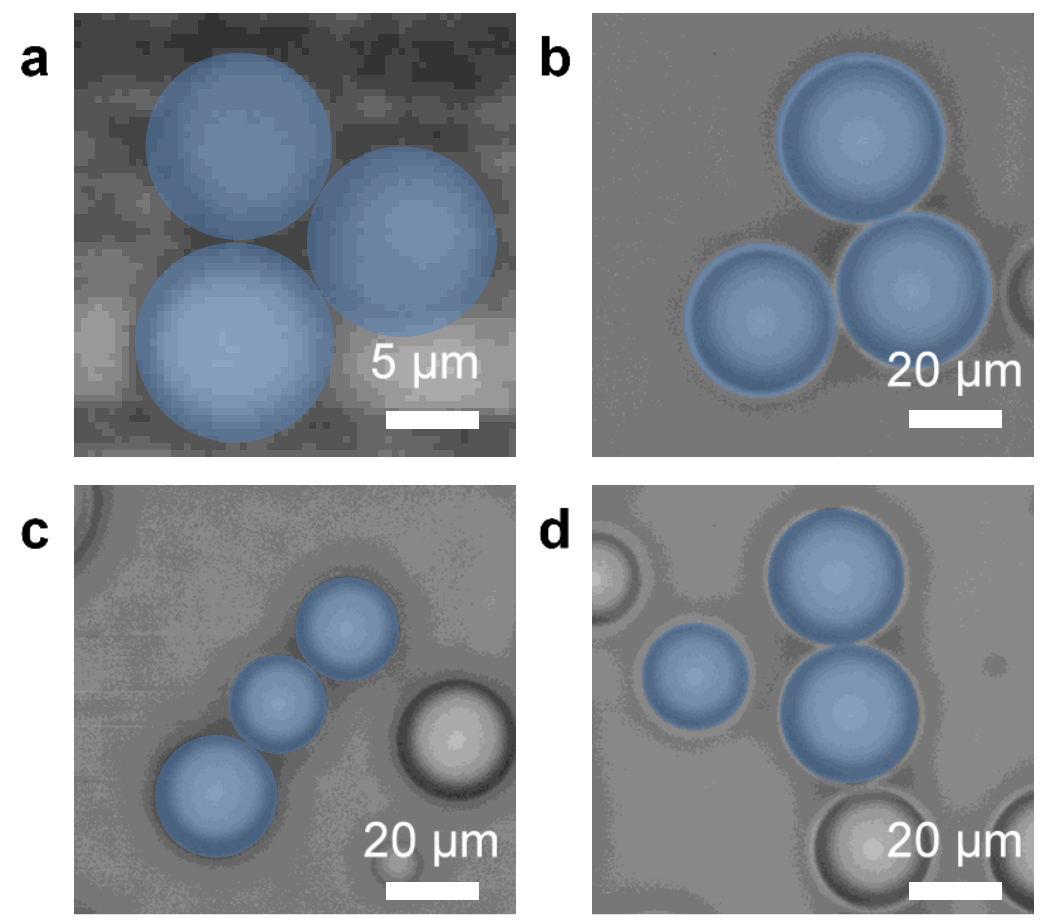

Figure S6. In-plane images of different droplet arrangements of initial coalescing droplets just prior to jumping where the number of initial droplets is 3 . Droplet pair contains (a) triangular, (b) L-shaped, (c) linear, and (d) spaced configurations. Coalescing droplets has been false-colored blue for clarity.

Figure $\mathrm{S} 7$ shows the jumping droplet speed, $U_{\mathrm{j}}$, as a function of departing droplet radius, $R_{\mathrm{j}}$, for three droplet coalescence with different initial droplet arrangements. The results show that the jumping droplet speed agrees well with inertial-capillary theory (dashed line), and that initial droplet arrangement is not correlated to the jumping droplet speed. Figure S8 shows the polar angle as a function of droplet size mismatch. After analysis of $>50$ jumping events having different configurations, no correlation was found between droplet arrangement prior to jumping, and polar angle deviation. 


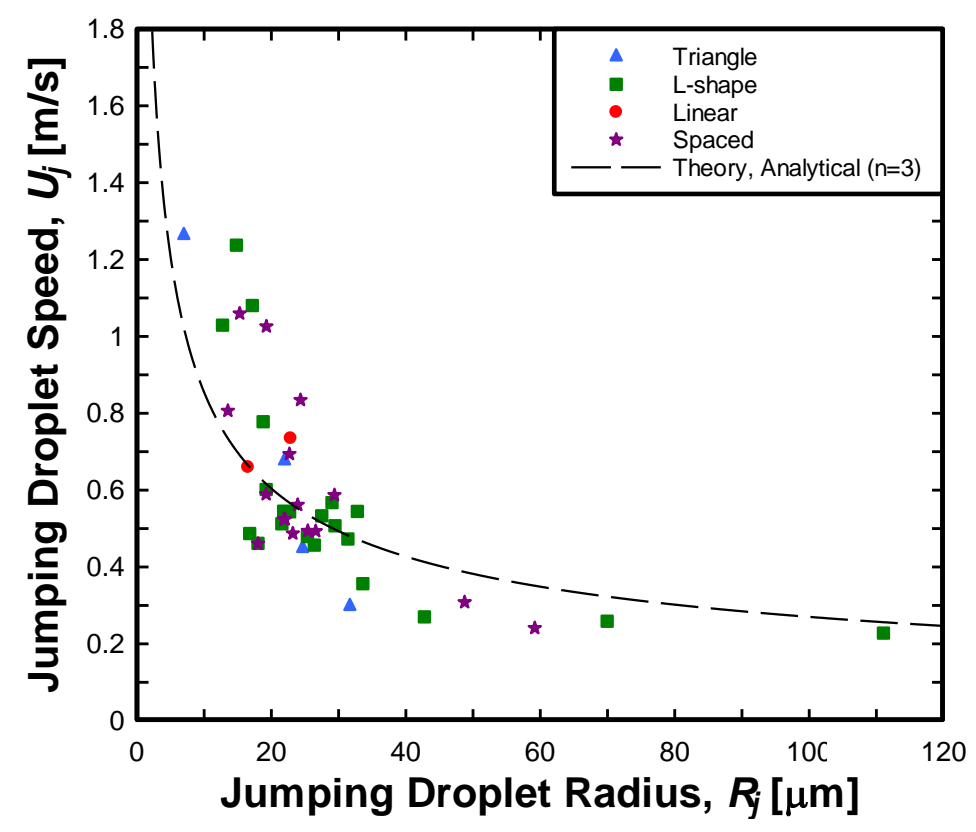

Figure S7. Jumping droplet speed, $U_{\mathrm{j}}$, as a function of departing droplet radius, $R_{\mathrm{j}}$, for three droplet coalescence.). The results show that initial droplet arrangement does not affect the jumping droplet speed for three droplet coalescence.

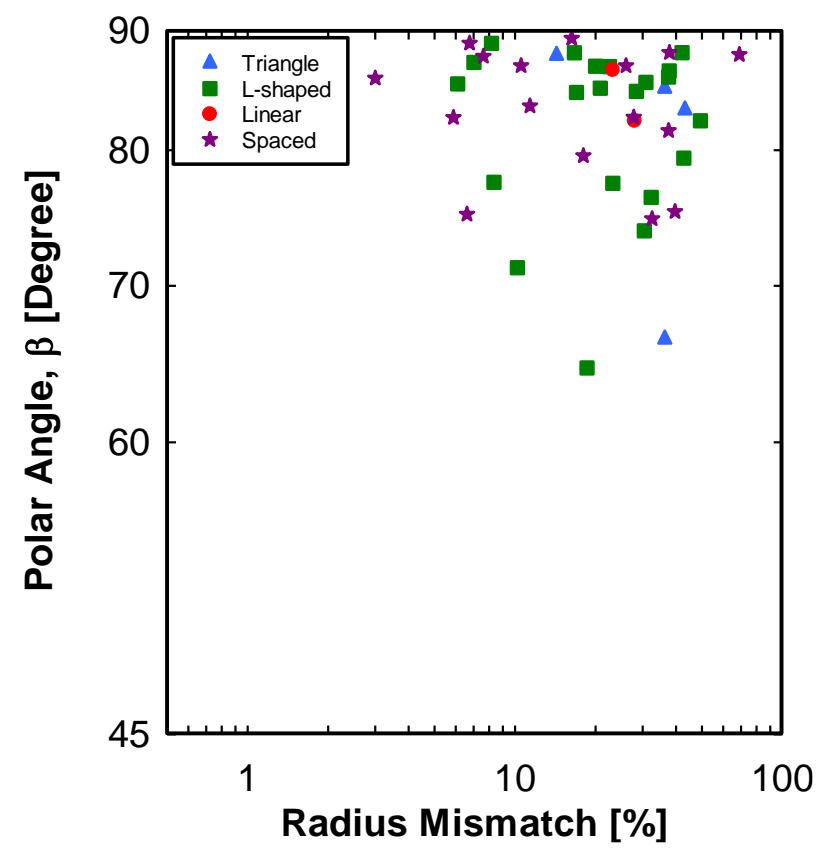

Figure S8. Polar angle as a function of droplet size mismatch for three droplet coalescence. The results show that initial droplet arrangement does not affect the jumping droplet speed for three droplet coalescence. 
To study the mechanism governing droplet polar angle deviation $\left(<80^{\circ}\right)$, we analyzed the droplet jumping videos to see if any particular droplet arrangement prior to droplet coalescence had any effect on departure polar angle. In particular, we analyzed all cases which resulted in polar angles $<80^{\circ}$ to see if we could identify any pattern in the initial droplet arrangement or coalescence behavior. Figure S9 shows the in-plane images of five particular coalescing droplet pairs just prior to jumping with (a) two, (b) three, (c) four, (d) four, and (d) five coalescing droplets resulting polar angles of $\beta=73^{\circ}, 71^{\circ}, 49^{\circ}, 55^{\circ}$, and $65^{\circ}$, respectively. The results of our analysis showed that initial droplet arrangement had no correlation to elevated polar angle deviation.
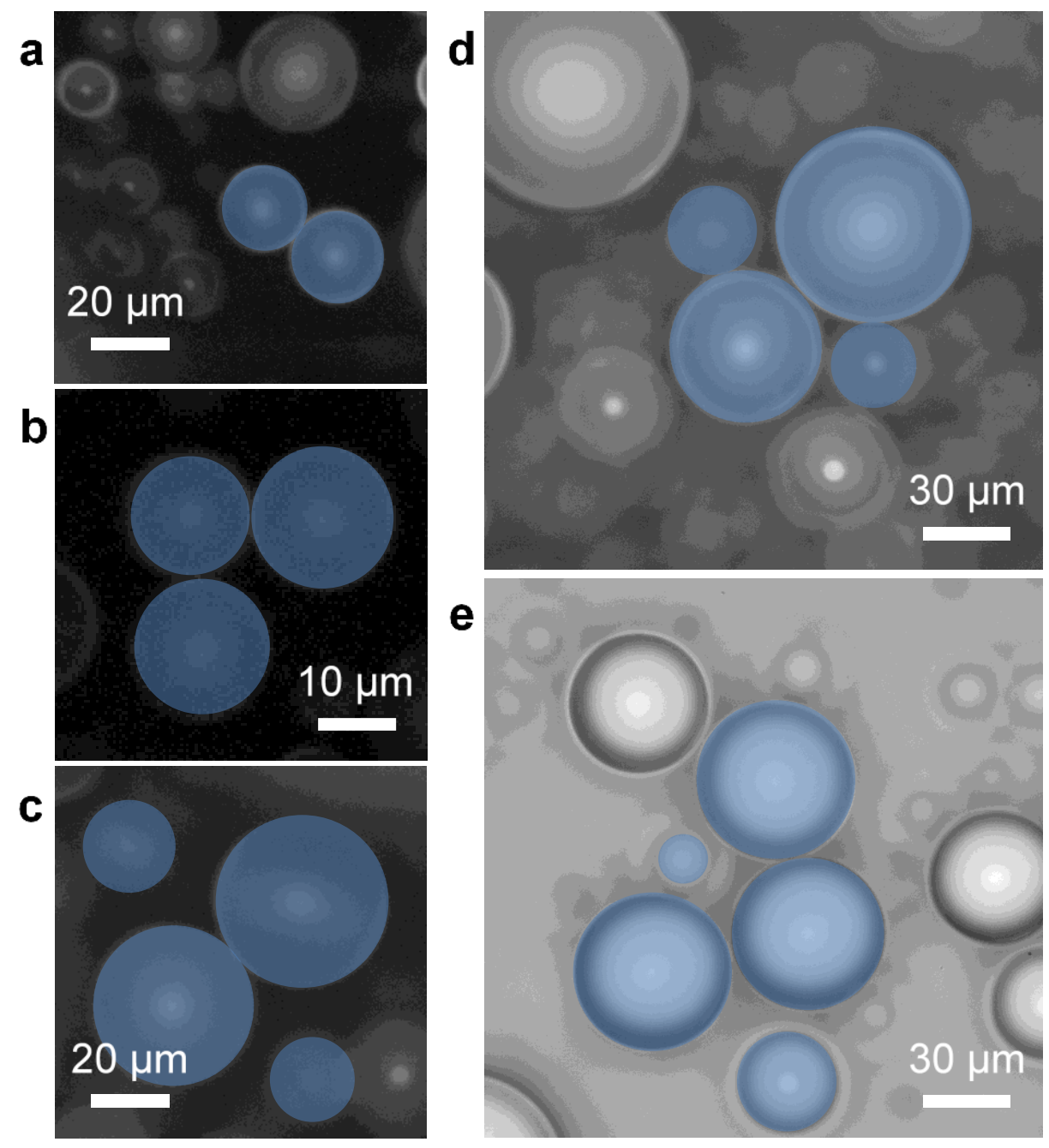

Figure S9. In-plane images of different initial droplet arrangements just prior to jumping with high polar angle deviation. Droplet pairs contain (a) 2, (b) 3, (c) 4, (d) 4, and (e) 5 coalescing droplets all departed with reduced polar angles $\beta=73^{\circ}, 71^{\circ}, 49^{\circ}, 55^{\circ}$, and $65^{\circ}$, respectively.

Droplets partaking in coalescence and jumping have been false-colored blue for clarity. 